\title{
XXXIV. The vapour pressures of binary liquid mixtures: Kinetic theory based on dieterici's equation
}

\section{Frank Tinker M.Sc.}

To cite this article: Frank Tinker M.Sc. (1916) XXXIV. The vapour pressures of binary liquid mixtures: Kinetic theory based on dieterici's equation , Philosophical Magazine Series 6, 32:189, 295-302, DOI: 10.1080/14786441608635574

To link to this article: http://dx.doi.org/10.1080/14786441608635574

曲 Published online: 08 Apr 2009.

Submit your article to this journal $₫$

ЏلI Article views: 2

Q View related articles $₫$ 
XXXIV. The Vapour Pressures of Binary Liquid Mirtures: Kinetic Theory based on Dieterici's Equation. By Frank Tinker, M.Sc.*

$\mathrm{N}$ a series of papers published at intervals during the last sixteen years $\dagger$, Dieterici has advanced a much more fundamental equation of state than the better known Tan der Waals' expression. 'The equation in question, viz.,

$$
p=\frac{\mathrm{RT}}{v-b} e^{-\frac{a}{v \mathrm{RT}}}, \quad \text {. . . . . }
$$

in which the constants $a$ and $b$ have a meaning similar to that given them by Van der Waals, is based on the following assumptions $\ddagger$ :-

(i.) The pressure $\pi$ within a fluid is given by the equation

$$
\pi(v-b)=\mathrm{RT}, . . . \quad . \quad . \quad[2]
$$

i. e., the product pressure $\times$ free space is given by the perfect gas equation; being independent of the size of the molecules, or of the forces between the molecules, and the same for all fluids at the same temperature.

(ii.) The pressure at the surface of the fluid is always less than that in the interior because of the inward pull exerted by the forces of cohesion. Assuming that the boundary pressure is proportional to the number of molecules which have kinetic energy enough to overcome the inward pull, Dieterici showed that this boundary pressure $(p)$ is related to the pressure within the fluid $(\pi)$ by the exponential equation

$$
p=\pi e^{-\frac{\mathrm{A}}{\mathrm{RT}},}
$$

where $A$ is the work done by the molecule in reaching the surface. Combination with equation [2] gives immediately

$$
p=\frac{\mathrm{RT}}{v-b} e^{-\frac{\mathrm{A}}{\mathrm{RT}}}, . .
$$

and making the further assumption that $A$ is proportional to the density of the fluid, and equal to $\frac{a}{v}$ where $a$ is a

* Communicated by Principal Sir O. J. Lodge, F.R.S.

† Ann. Phys. u. Chem. xi. p. 700 (1899); Ann. der Physik [4] v. p. 51 (1901); ibid. xxv. p. 269 (1908); ibid. xxxv. p. 220 (1911).

$t$ The assumptions are also similar to Van der Waals', but are given a different quantilative expression. The two equations become identical at low and medium pressures. 
constant for the substance in question, the equation [4] becomes identical with equation [1].

Dieterici himself has shown that his equation gives excellent results when applied to critical data in the usual way. Recently others also have taken up the development of the subject. In particular McDougall has lately demonstrated * that the pressure of the saturated vapour over a liquid is also given by the equation to a high degree of aecuracy.

In view, therefore, of the interest which has been revived in the causes for the deviations of the vapour pressures of binary mixtures from the well-known law of admixture in molecular proportions, and on account of the importance of the question to the subject of osmotic pressure, it seemed that it would be of interest to extend the application of Dieterici's equation to such mixtures also. Van Laar has already studied the subject from the thermodynamic side. It will be seen from the treatment which follows that Dieterici's equation leads to expressions for the partial and total vapour pressures identical in form with those obtained by Van Laar with the aid of the thermodynamic potential; but that the kinetic treatment enables the subject to be developed further, and gives a very simplo relationship between the variation of the vapour pressure of the mixture from the theoretical, and the variation of the latent heat of vaporization from the theoretical value calculated by the mixture rule.

(a) Relation between the Partial and Total Liquid Pressures in a binary mixture and the relative molecular concentrations of the two components $\dagger$.

Let $\mathrm{X}$ and $\mathrm{Y}$ be the two components of the liquid mixture, and let $\mathrm{N}$ molecules of $\mathrm{X}$ be mixed with $n$ molecules of $\mathrm{Y}$.

* Journ. Amer. Chem. Soc. xxxviii. p. 528 (1916). Mr. McDougall shows that

$$
p_{s}=\frac{\mathrm{RT}}{v_{1}-b} e^{-\frac{a}{v_{1} R \overline{\mathrm{T}}}}=\frac{\mathrm{RT}}{v_{2}-b} e^{-\frac{a}{v_{2} \mathrm{RT}}}=\frac{\mathrm{RT}}{b} e^{-\frac{a}{2 b \mathrm{R} T}},
$$

where $v_{1}$ and $v_{2}$ refer to the specific volumes of the liquid and vapour respectively, and where $a$ and $b$ have the values given by the equations

$$
\frac{1}{b}=\frac{1}{v_{1}}+\frac{1}{v_{2}} ; \quad a=\frac{2 v_{1} v_{2}}{v_{1}+v_{2}} \mathrm{RT} \log e \frac{v_{2}}{v_{1}} .
$$

$\dagger$ In what follows I use the term liquid pressure $(\pi)$ to denote the bombardment pressure exerted by the liquid molecules on either side of a plane of unit area placed anywhere within the liquid. It is of course different from the internal or intrinsic pressure due to cohesion. In another place ('Nature,' vol. xcvii. p. 122 (1916)) I have glso called it the "diffusion pressure" to distinguish it from the latter. 
Also let $\quad \pi_{1}=$ liquid pressure of pure $\mathrm{X}$,

$\begin{aligned} \pi_{2} & =\text {, " } \pi_{1}{ }^{\prime} \text { and } \pi_{2}^{\prime}=\text { partial liquid pressures of } \mathrm{X} \text { and } \mathrm{Y} \text { in }\end{aligned}$ the mixture,

$\pi=\pi_{1}{ }^{\prime}+\pi_{2}{ }^{\prime}=$ total liquid pressure in mixture,

$\mathrm{V}_{1}$ and $\mathrm{V}_{2}=$ molecular volumes of pure $\mathrm{X}$ and $\mathrm{Y}$,

$\mathrm{V}_{1}{ }^{\prime}$ and $\mathrm{V}_{2}{ }^{\prime}=\quad, \quad, \quad$ of $\mathrm{X}$ and $\mathrm{Y}$ in the mixture.

In general, $V_{1}^{\prime}$ and $V_{2}^{\prime}$ will be slightly different from $V_{1}$ and $V_{2}$ owing to the small volume changes which take place on mixing.

Consider first the partial liquid pressure $\pi_{1}{ }^{\prime}$ of the component $X$. Before the $N$ molecules of $X$ are introduced into the mixture, the liquid pressure of the pure $\mathrm{X}$ is given by the relation

$$
\pi_{1}=\frac{\mathrm{RT}}{\mathrm{N}\left(\mathrm{V}_{1}-b_{1}\right)}, \quad \cdot \quad \cdot \quad . \quad . \quad .
$$

where the constant $R$ refers to the mass of $N$ molecules. Now add the $n$ molecules of $\mathrm{Y}$ to the $\mathrm{N}$ molecules of $\mathrm{X}$. We have, volume of free space in mixture

$$
=\mathrm{N}\left(\mathrm{V}_{1}^{\prime}-b\right)+n\left(\mathrm{~V}_{2}^{\prime}-b_{2}\right) \text {. }
$$

Hence partial pressure $\pi_{1}{ }^{\prime}$ of component will be given by the equation

$$
\pi_{1}^{\prime}=\frac{\mathrm{RT}}{\mathrm{N}\left(\mathrm{V}_{1}^{\prime}-b_{1}\right)+n\left(\mathrm{~V}_{2}^{\prime}-b_{2}\right)}=\frac{\mathrm{RT}}{(\mathrm{N}+n)\left(\mathrm{V}_{1}^{\prime}-b_{1}\right)},
$$

since the two "free spaces" $\left(V_{1}^{\prime}-b_{1}\right)$ and $\left(V_{2}^{\prime}-b_{2}\right)$, being in the same mixture, are equal to one another.

Combining equations [5] and [6] we obtain

$$
\frac{\pi_{1}^{\prime}}{\pi_{1}}=\frac{\mathrm{N}}{\mathrm{N}+n} \cdot \frac{\mathrm{V}_{1}-b_{1}}{\mathrm{~V}_{1}^{\prime}-b_{1}}=\frac{\mathrm{N}}{\mathrm{N}+n}\left(1-\epsilon_{1}\right) \text { approx., . }
$$

where $\epsilon_{1}$ denotes the fractional increase in the "free space" of the molecule of $\mathrm{X}$ by the mixing.

Similarly

$$
\frac{\pi_{2}^{\prime}}{\pi_{3}}=\frac{n}{\mathrm{~N}+n}\left(1-\varepsilon_{3}\right)
$$

If instead of $\frac{\mathrm{N}}{\mathrm{N}+n}$ and $\frac{n}{\mathrm{~N}+n}$ we substitute the molar fractions $x$ and $(1-x)$ in the usual way, equations [7] and [8] becom $\theta$

$$
\begin{aligned}
& \pi_{1}^{\prime}=\pi_{1} x\left(1-\epsilon_{1}\right), \quad \text {. . . . . . . }[9] \\
& \pi_{2}{ }^{\prime}=\pi_{2}(1-x)\left(1-\epsilon_{2}\right) . \quad \text {. . . . }[10]
\end{aligned}
$$

Phil. Mag. S. 6. Vol. 32. No. 189. Sept. 1916. 
We may usually neglect the volume changes of liquids on mixing, so that $\epsilon_{1}$ and $\epsilon_{2}$ are practically zero. In this case, the total liquid pressure $\pi$ becomes

$$
\pi=\pi_{1}{ }^{\prime}+\pi_{2}{ }^{\prime}=\pi_{1} x+\pi_{2}(1-x), \quad . \quad \cdot[11]
$$

Hence when there are no volume changes on mixing, the total liquid pressure can be calculated from the two partial liquid pressures by the law of admixture in molecular proportions. For the partial liquid pressure of each component is then proportional to its molar fraction. If, however, there is a volume change, the law is only approximately accurate. But the ratio $\frac{V_{1}-b_{1}}{V_{1}^{\prime}-b_{1}}=\left(1-\epsilon_{1}\right)$ will in general be so nearly equal to unity that there will be an appreciable deviation from the mixture law only in very abnormal cases.

(b) Relation between the Partial and Total Vapour Pressures in a binary mixture and the relative molecular concentrations of the two components.

We can now apply Dieterici's equation proper to the questions in hand.

Let $p_{1}$ and $p_{2}=$ vapour pressures of pure components $\mathrm{X}$ $p_{1}^{\prime}$ and $p_{2}^{\prime}=, \quad, \quad$ of $\mathrm{X}$ and $\mathrm{Y}$ in mixture, $p=p_{1}{ }^{\prime}+p_{2}^{\prime}=$ total vapour pressure of mixture,

$A_{1}$ and $A_{2}=$ work done when a molecule of $X$ or $Y$ is evaporated from the pure liquids, $A_{1}{ }^{\prime}$ and $A_{2}{ }^{\prime}=$ work done when a molecule of $X$ or $Y$ is evaporated from the mixture.

$\mathrm{N}, n, x$ have same values as before.

From equation [3] we have

$$
\begin{aligned}
& p_{1}=\pi_{1} e^{-\frac{A_{1}}{R T}}, \\
& p_{1}^{\prime}=\pi_{1}^{\prime} e^{-\frac{A_{1}}{\mathrm{RT}}} .
\end{aligned}
$$

Hence

$$
\frac{p_{1}^{\prime}}{p_{1}}=\frac{\pi_{1}^{\prime}}{\pi_{1}} e^{-\frac{\left(\mathbf{A}_{1}^{\prime}-\mathbf{A}_{2}\right)}{\mathrm{RT}}}
$$

Combining this equation with equation [9] and neglecting $\epsilon$, we have

$$
p_{1}^{\prime}=p_{1} x e^{-\frac{\left(A_{1}^{\prime}-A_{1}\right)}{\mathrm{RT}}} \cdot . . . \quad . .
$$


Similarly

$$
p_{2}^{\prime}=p_{2}(1-x) e^{-\frac{\left(\mathbf{A}_{2}^{\prime}-\mathbf{A}_{2}\right)}{\mathrm{RT}}}
$$

and

$$
p=p_{1}^{\prime}+p_{2}^{\prime}=p_{1} x e^{-\frac{\left(\mathbf{A}_{1}^{\prime}-\mathbf{A}_{1}\right)}{\mathrm{RT}}}+p_{2}(1-x) e^{-\frac{\left(\mathbf{A}_{2}^{\prime}-\mathbf{A}_{2}\right)}{\mathrm{RT}}}
$$

The last equation is of the same form as the equation Van Laar has obtained for the total vapour pressure of a binary mixture on thermodynamic grounds. Van Laar's expression is

$$
p=p_{1} x e^{-\frac{\left(\mu_{1}^{\prime}-\mu_{1}\right)}{\mathrm{RT}}}+p_{2}(1-x) e^{-\frac{\left(\mu_{2}^{\prime}-\mu_{2}\right)}{\mathrm{RT}}},
$$

where $\mu_{1}$ and $\mu_{2}$ are the thermodynamic potentials of the two components*. form

Equation [13] can also be written in the more convenient

$$
p=p_{\mathrm{L}} x e^{-\frac{\partial A_{1}}{\mathrm{RT}}}+p_{2}(1-x)^{-\frac{\partial A_{2}}{R T}}, \quad \text {. . }
$$

where $\partial A_{1}$ and $\partial A_{2}$ represent the increase in the work done by evaporating a molecule of $\mathrm{X}$ or $\mathrm{Y}$ from the mixture over the work done when the molecule is evaporated from the pure liquid.

Furthermore, it will be seen that $\partial A_{1}$ and $\partial A_{2}$ are practically identical with the excess of the molecular latent heats of vaporization $\partial \mathrm{L}_{1}$ and $\partial \mathrm{L}_{2}$ of the two components in the mixture over their molecular latent heats in the pare state. Hence we may write $\dagger$

$$
p=p_{1} x e^{-\frac{\partial \underline{L}_{1}}{R T}}+p_{2}(1-x) e^{-\frac{\partial \mathrm{r}_{2}}{\mathrm{RT}}} . \quad \text {. . }
$$

We may develop equation [15] still further. Expanding $e^{-\frac{\partial \mathrm{L}_{1}}{\mathrm{RT}}}$ and $e^{-\frac{\partial \mathrm{L}_{2}}{\mathrm{RT}}}$ we have, since $\partial \mathrm{L}_{1}$ and $\partial \mathrm{L}_{2}$ are small,

$$
\begin{aligned}
p & =p_{1} x\left(1-\frac{\partial \mathrm{L}_{1}}{\mathrm{RT}}\right)+p_{2}(1-x)\left(1-\frac{\partial \mathrm{L}_{2}}{\mathrm{RT}}\right) \\
& =p_{1} x+p_{2}(1-x)-\frac{1}{\mathrm{R}^{\top} \mathrm{T}}\left\{p_{1} x \partial \mathrm{L}_{1}+p_{2}(1-x) \partial \mathrm{L}_{2}\right\} .
\end{aligned}
$$

Now if $p_{0}$ is the vapour pressure of the mixture calculated

* Zeit. Phys. Chem. Ixxii. p. 723 (1910) ; ibid. Ixxxiii. p. 599 (1913); and other papers.

+ Equation [15] indicates that $\partial \mu=\partial \mathrm{L}$, or that as regards the vaporization of liquids, increase in thermodynamic potential is equivalent to increase in latent heat of vaporization. 
according to the law of admixture in molecular proportions, and if $L_{s}$ denotes the molecular latent heat of vaporization of the perfect mixture*, we have

$$
p_{0}=p_{1} x+p_{2}(1-x)
$$

and

$$
p_{0} \mathrm{~L}=p_{1} x \mathrm{~L}_{1}+p_{2}(1-x) \mathrm{L}_{2}, \quad . \quad .
$$

since the two components evaporate in the ratio of their partial pressures.

Hence also

$$
p_{1} x \partial \mathrm{L}_{1}+p_{2}(1-x) \partial \mathrm{L}_{2}=p_{0} \partial \mathrm{L}
$$

Consequently

$$
\begin{aligned}
p & =p_{1} x+p_{2}(1-x)-p_{0} \frac{\partial \mathrm{L}}{\mathrm{R}^{\prime} \mathrm{T}} \\
& =p_{0}-p_{0} \frac{\partial \mathrm{L}}{\mathrm{R}^{\prime} \mathrm{T}},
\end{aligned}
$$

i. e.

$$
p=p_{0}\left(1-\frac{\partial \mathrm{L}}{\mathrm{RT}}\right) ; \quad \cdot \quad \cdot \quad \cdot \quad .
$$

or in words, "the total vapour pressure of a binary mixture is equal to the vapour pressure calculated from the law of admixture in molecular proportions, multiplied by a factor $\left(1-\frac{\partial \mathrm{L}}{\mathrm{R}^{\prime} \mathrm{L}}\right)$, where $\partial \mathrm{L}$ is the excess of the molecular latent heat of vaporization of the mixture over the theoretical value calculated from the mixture rule."

This simple relationship shows that the variation of the total vapour pressure from the straight line law is determined almost entirely by abnormalities in the latent heat of vaporization $\dagger$.

Proceeding from equation [17] we have three possible cases :-

(i.) If the actual molecular heat of vaporization of the

* The actual inolecular latent heat of the mixture would be the heat of evaporation or condensation of one gm. molecule of vapour, i.e. the heat required to produce $22 \cdot 2$ litres of the mixed vapour at N.T.P. The theoretical value $\mathrm{L}$ is of course calculated from equation [16].

+ Volume changes on mixing will of course have a slight effect on the vapour pressure also. Allowing for these, equation [17] becomes

$$
p=p_{0}\left(1-\frac{\partial \mathrm{L}}{\mathrm{RT}}\right)-\left(p_{1}^{\prime} \epsilon_{1}+p_{2}{ }^{\prime} \boldsymbol{\epsilon}_{2}\right) .
$$


mixture is equal to the theoretical value $(\partial \mathrm{L}=0)$, the vapour-pressure curve is a straight line (Diag. I.).

Diagram I.

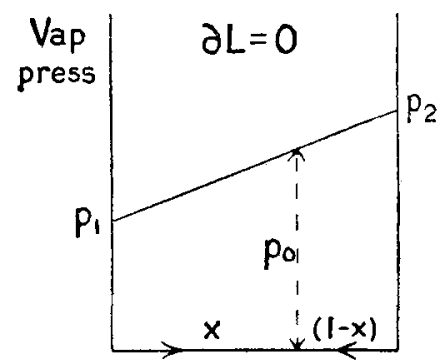

Diagram II.

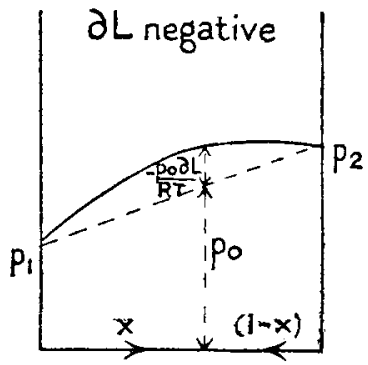

Molar fractions of two components.

(ii.) If the molecular latent heat of vaporization is less than the theoretical (i.e. $\partial \mathrm{L}$ is negative) the vapour pressure is greater than the theoretical (Diag. II.).

(iii.) If the molecular heat of vaporization is greater than the theoretical (i.e. $\partial \mathrm{L}$ is positive) the vapour pressure is less than the theoretical (Diag. III.).

Diagram III.

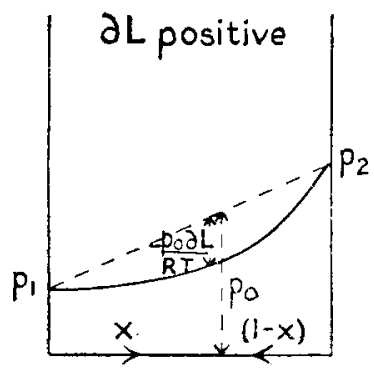

Diagram IV.

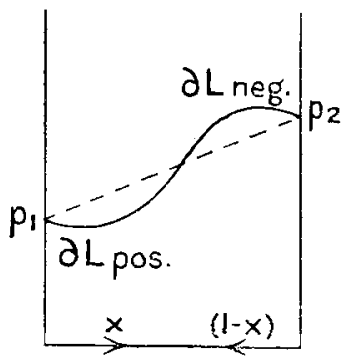

If, as the relative molecular concentration of the two components is varied, the value of $\partial L$ changes from positive to negative or vice versa, the curve will cross over the diagonal (as in Diag. IV.). In this case $\partial \mathrm{L}$ is at first positive, and then changes over to negative.

As yet there have been no measurements made by which the above equation [17] can be subjected to a quantitative test. But its general soundness can be shown indirectly from surface-tension data. It is well known that there is a 
very close connexion between the latent heat of vaporization of a liquid and its surface-tension, liquids having a high latent heat, for instance, also having a high surface-tension *. Abnormally high vapour pressures are therefore to be associated also with abnormally low surface-tensions, and vice versa. F. P. Worley has already advanced this view on experimental grounds $\dagger$. Corresponding to the three cases just given for the differences in latent heats, this investigator has given the three following rules for the connexion between the vapour pressures of binary mixtures and their surfacetensions :-

"(i.) If at any given temperature the vapour pressures of mixtures of two liquids agree with the values calculated by the rule of admixture in molecular proportions, the surfacetensions of the mixtures agree with those calculated by the formula

$$
S=V_{1} S_{1}+V_{2} S_{2} \ddagger \text {. }
$$

"(ii.) If the vapour pressures are greater than those calculated, then the surface-tensions are less than those. calculated.

" (iii.) If the vapour pressures are less than those calculated, the surface-tensions are greater than those calculated."

By comparing his own and other surface-tension curves with the vapour-pressure curves of Zawidski and others, Worley has shown that every pair of liquids so far investigated comes under the rules laid down. Mixtures of benzene and ethylene dichloride, for instance, form perfect mixtures and obey the first rule; carbon bisulphide and acetone, benzene and carbon tetrachloride, ether and carbon bisulphide obey the second rule; whilst in the same way, water and the alcohols, and pyridine and acetic acid obey the third.

* So much can be deduced from the Laplace Theory of Capillarity. Also Walden has given the following empirical formula for this relationship, based on a large number of experimental data :

$$
\mathrm{L}=3.64 v \gamma,
$$

where $\mathrm{L}=$ latent heat per gm. in calories ; $v=$ sp. vol. in c.c. ; $\gamma=$ surfacetension in dynes $/ \mathrm{cm}$.

† Journ. Chem. Soc. Trans. cv. [1] p. 273 (1914).

$\mp V_{1}$ and $V_{2}$ are the volumes of the two liquids expressed fractionally. 